\title{
Caractérisation Hydrodynamique et Qualité des eaux de l'aquifère à nappe libre du bassin versant du Nkié (Yaoundé-Cameroun)
}

\author{
Abdou Nasser Ngouh, \\ Institut National de Cartographie, Laboratoire de Traitement d'Image pour la \\ Stériorestitution, Département des Sciences de la Terre et de l'Univers, \\ Université de Yaoundé I, Cameroun
}

\section{Amidou Kpoumie,}

Laboratoire de Géosciences et Developpement Durable, Département des

Sciences de la Terre, Faculté des Sciences,

Université de Maroua, Cameroun

Ghislain Nyembe Etame,

Institut National de Cartographie, Laboratoire de Traitement d'Image pour la

Stériorestitution, Département de Géographie,

Université de Maroua, Cameroun

Aloysious Kohtem Lebga,

Institut National de Cartographie,

Laboratoire de Traitement d'Image pour la Stériorestitution, Cameroun

Esse Ndjeng,

Institut National de Cartographie, Cameroun

Jules Remy Ndam Ngoupayou,

Département des Sciences de la Terre et de l'Univers,

Université de Yaoundé I, Cameroun

Doi:10.19044/esj.2020.v16n15p281 URL:http://dx.doi.org/10.19044/esj.2020.v16n15p281

\begin{abstract}
Between September 2017 and August 2018, a study aimed at carrying out a physical characterization, understanding the hydrodynamic behavior and evaluating the quality of the waters of the unconfined aquifer of the Nkié watershed was carried out. The field and laboratory work carried out jointly consisted of the fortnightly monitoring of the hydraulic structures previously identified, in situ measurements of permeability, soil and water sampling for analysis of particle size and physico-chemical and bacteriological parameters. At the end of this work, the particle size analysis of the watershed soils reveals a reduction in the particle size from the base to the top of the profile. The weight carryover of the different soil levels makes it
\end{abstract}


possible to distinguish gravelly sands, sands, sandy gravels and sandy clays. These lithological formations have permeabilities which vary between $1.8 .10^{-6} \mathrm{~m} \cdot \mathrm{s}^{-1}$ and $5.92 .10^{-5} \mathrm{~m} \cdot \mathrm{s}^{-1}$, thus illustrating that they are little to fairly permeable. The daily flow rates of the sources vary between $0.691 . \mathrm{s}^{-1}(2.5$ $\left.\mathrm{m}^{3} \cdot \mathrm{h}^{-1}\right)$ and $0.061 . \mathrm{s}^{-1}\left(0.22 \mathrm{~m}^{3} \cdot \mathrm{h}^{-1}\right)$ in the dry season (D.S) and between 0.86 $1 . \mathrm{s}^{-1}\left(3.1 \mathrm{~m}^{3} \cdot \mathrm{h}^{-1}\right)$ and $0.081 . \mathrm{s}^{-1}\left(0.3 \mathrm{~m}^{3} \cdot \mathrm{h}^{-1}\right)$ in the rainy season (R.S). The sources of larger flows would be located on the main drainage axes corresponding to the main fracturing axes (N10-30, N40-50, N80-100, N110120). Analysis of the piezometric maps reveals a convergence of the streamlines towards the Nkié stream, which is the main drainage axis of the unconfined aquifer. The established water balance shows that the alterite reservoir is recharged from April to November under the action of excess rainfall and is emptied from December to March during low water. Thus, the hydrodynamic behavior of the aquifer is closely linked to rainfall fluctuations. The spring waters are acidic and not very mineralized. These are waters of the sodium and potassium chloride type. The noted presence of nitrates, fecal coliforms and fecal streptococci whose concentrations are above the toxicity limits defined by the WHO (2011) induce bacteriological and chemical pollution of these waters. They therefore require prior treatment for this purpose before any consumption.

Keywords: Nkié Watershed, Yaoundé-Cameroon, Physical And Hydrodynamic Characterisation, Water Quality, Unconfined Aquifer 


\title{
Hydrodynamic Characterization and Water Quality of the Aquifer of the Nkié Watershed (Yaoundé-Cameroon)
}

\begin{abstract}
Abdou Nasser Ngouh, Institut National de Cartographie, Laboratoire de Traitement d'Image pour la Stériorestitution, Département des Sciences de la Terre et de l'Univers, Université de Yaoundé I, Cameroun
\end{abstract}

Amidou Kpoumie,

Laboratoire de Géosciences et Developpement Durable, Département des

Sciences de la Terre, Faculté des Sciences,

Université de Maroua, Cameroun

Ghislain Nyembe Etame,

Institut National de Cartographie, Laboratoire de Traitement d'Image pour la

Stériorestitution, Département de Géographie,

Université de Maroua, Cameroun

Aloysious Kohtem Lebga,

Institut National de Cartographie,

Laboratoire de Traitement d'Image pour la Stériorestitution, Cameroun

Esse Ndjeng,

Institut National de Cartographie, Cameroun

Jules Remy Ndam Ngoupayou,

Département des Sciences de la Terre et de l'Univers,

Université de Yaoundé I, Cameroun

\section{Résumé}

Entre septembre 2017 et août 2018, une étude visant à faire une caractérisation physique, à comprendre le comportement hydrodynamique et à évaluer la qualité des eaux de l'aquifère à nappe libre du bassin versant du Nkié a été menée. Les travaux de terrain et de laboratoire menés conjointement ont consisté au suivi bimensuel des ouvrages hydrauliques préalablement identifiées, aux mesures in situ de la perméabilité, aux échantillonnages des sols et des eaux pour analyses de la granulométrie et des paramètres physico chimiques et bactériologiques. A l'issue de ces travaux, l'analyse granulométrique des sols du bassin versant révèle une diminution de la taille des particules de la base vers le sommet du profil. Le report pondéral des différents niveaux de sols, permet de distinguer les sables 
graveleux, les sables, les graviers sableux et les argiles sableuses. Ces formations lithologiques ont des perméabilités qui varient entre $1,8 \cdot 10^{-6} \mathrm{~m} \cdot \mathrm{s}^{-1}$ et $5,92 \cdot 10^{-5} \mathrm{~m} \cdot \mathrm{s}^{-1}$, illustrant ainsi qu'elles sont peu à assez perméables. Les débits journaliers des sources varient entre $0,691 \cdot \mathrm{s}^{-1}\left(2,5 \mathrm{~m}^{3} \cdot \mathrm{h}^{-1}\right)$ et $0,061 \cdot \mathrm{s}^{-1}$ $\left(0,22 \mathrm{~m}^{3} \cdot \mathrm{h}^{-1}\right)$ en saison sèche (S.S) et entre $0,861 . \mathrm{s}^{-1}\left(3,1 \mathrm{~m}^{3} \cdot \mathrm{h}^{-1}\right)$ et $0,081 . \mathrm{s}^{-1}$ $\left(0,3 \mathrm{~m}^{3} \cdot \mathrm{h}^{-1}\right)$ en saison pluvieuse (S.P). Les sources de plus grands débits seraient situées sur les principaux axes de drainage correspondant aux axes principaux de fracturation (N10-30, N40-50, N80-100, N110-120). L'analyse des cartes piézométriques révèle une convergence des lignes de courant d'eau vers le cours d'eau Nkié qui en est le principal axe de drainage de l'aquifère à nappe libre. Le bilan hydrique établi, montre que le réservoir d'altérites se recharge d'avril à novembre sous l'action de l'excédent pluviométrique et se vide de décembre à mars lors de l'étiage. Ainsi, le comportement hydrodynamique de la nappe est étroitement lié aux fluctuations pluviométriques. Les eaux de sources sont acides et peu minéralisées. Ce sont les eaux de type chloruré sodique et potassique. La présence remarquée des nitrates, des coliformes fécaux et des streptocoques fécaux dont les concentrations sont supérieures aux limites de toxicité définies par l'OMS (2011) induisent une pollution bactériologique et chimique de ces eaux. Elles nécessitent donc à cet effet un traitement préalable avant toute consommation.

Mots clés : Bassin Versant Du Nkié, Yaoundé-Cameroun, Caractérisation Physique, Hydrodynamique, Qualité Des Eaux, Aquifère À Nappe Libre

\section{Introduction}

L'eau est une ressource naturelle importante, qui constitue le noyau du système écologique (Selvam et al., 2013). Elle joue un rôle extrêmement important pour les êtres vivants notamment dans le développement socioéconomique et les écosystèmes (An et al., 2014 ; Ma et al., 2014 ; Eshtawi et al., 2016). Au cours du Sommet Mondial sur le Développement Durable (SMDD) tenu à Johannesburg en 2002, un constat global s'est dégagé à savoir l'amenuisement progressif des ressources en eau disponibles et mobilisables. Cette diminution est liée à une forte croissance démographique, au développement industriel, urbain et agricole auxquels s'ajoutent les aléas de la variabilité et du changement climatiques qui entrainent une augmentation sans cesse des besoins en eau (MINEE-GWP, 2009). La ville de Yaoundé (Capitale politique du Cameroun), à l'instar de la majorité des villes subsahariennes a été marquée au cours des deux dernières décennies par de grandes mouvances notamment sur le plan démographique. Cette ville a une superficie d'environ $310 \mathrm{~km}^{2}$, une densité de population de 44,9 habitants $/ \mathrm{km}^{2}$, un taux de croissance annuel de $5,7 \%$ et une population 
d'environ 1.817.524 habitants (BUCREP, 2010). On peut donc estimer actuellement la population de Yaoundé à plus de 2800000 habitants. Cette forte croissance démographique s'est accompagnée d'une forte demande en eau potable et en services d'assainissement (Kouam et al., 2006). Cette ville est alimentée en eau potable par captage du fleuve Nyong à partir de la station d'Akomnyada près de Mbalmayo. En effet, la CAMWATER (Cameroon Water Utilities Coorporation) estime les besoins journaliers actuels en eau potable de Yaoundé a plus de $250000 \mathrm{~m}^{3} / \mathrm{j}$ et ne peut fournir en retour qu'environ $110000 \mathrm{~m}^{3} / \mathrm{j}$, d'où un déficit journalier de $140000 \mathrm{~m}^{3} / \mathrm{j}$ (-56 \%) (MINEE-GWP, 2009). Le réseau de distribution de la CAMWATER alimente seulement $54 \%$ de la population urbaine (LESEAU, 1998 ; Djeuda et al., 1998 ; Djeuda et al., 2001 ; Tanawa et al., 2003). Ce déficit d'eau potable couplé aux coupures intempestives et au pouvoir d'achat faible pour pouvoir se brancher au réseau d'adduction d'eau potable, amène le reste de la population à utiliser les eaux de pluie, de marigots et de nappes d'aquifères de subsurface (puits et sources) dont les qualités et les caractéristiques physiques et hydrodynamiques sont très peu connues. Ainsi le problème d'approvisionnement en eau potable se pose alors avec acuité. Malgré le fait que le droit à l'eau ait été assimilé au niveau international à un droit de l'homme (UNESCO, 2003), la majorité de ces citadins vivent dans des quartiers précaires dépourvus d'eau courante et de systèmes d'assainissement adéquats, ce qui constitue des menaces pour leur santé. Le choix du bassin versant du Nkié, qui draine partiellement la commune d'arrondissement de Yaoundé $4^{\text {ème }}$ au Cameroun, souscrit à cette logique et les travaux entrepris dans ce bassin afin de contribuer à la connaissance et à la gestion optimale des ressources en eau souterraine de la ville de Yaoundé. L'objectif de ce travail est d'évaluer la qualité des eaux (physico-chimique et bactériologique) et de comprendre le fonctionnement hydrodynamique de l'aquifère à nappe libre du bassin versant du Nkié qui draine la partie sudouest du grand bassin versant du Mfoundi de la ville de Yaoundé Cameroun.

\section{Présentation du site d'étude}

Le bassin versant de Nkié avec une superficie de $5,17 \mathrm{~km}^{2}$ et un périmètre de 14,66 km est situé au Sud-Est du bassin versant de Mfoundi : principal cours d'eau qui draine Yaoundé la capitale politique camerounaise. Ce bassin s'étend entre $03^{\circ} 48^{\prime} 38^{\prime \prime}$ et $03^{\circ} 50^{\prime} 55^{\prime \prime}$ de latitude Nord, 011 $31^{\circ} 10^{\prime \prime}$ et $011^{\circ} 32^{\prime} 52^{\prime \prime}$ de longitude Est (Figure 1).

Sur le Plan administratif, ce bassin couvre partiellement l'arrondissement de Yaoundé IV et draine plusieurs quartiers parmi lesquels Ekounou, Ekié, Nkol-kié, Mvan et Ahala I. 
Le bassin versant du Nkié présente un relief caractérisé par l'alternance des collines peu étendues à pentes irrégulières et à vallées drainées par le cours d'eau principal Nkié et ses affluents. Ainsi, l'agencement du relief révèle une subdivision du site en plusieurs unités géomorphologiques avec des altitudes maximales de $785 \mathrm{~m}$ dans la partie nord et minimale de $660 \mathrm{~m}$ dans la partie sud (Figure 1).

Le substratum géologique est constitué de roches métamorphiques (gneiss, migmatites) d'âge précambrien (Nzenti et al., 1988 ; Nzenti et Tchoua, 1996). Les faciès rencontrés sont principalement des gneiss à grenat et plagioclases, des gneiss à grenat et à disthène, les pyriclasites, les pyribolites et les pyroxénites à magnétite. Sur ces roches se développent les sols ferralitiques et hydromorphes (Ekodeck, 1984 ; Onguene Malla, 1993 ; Ekodeck et Kamgang-Kabeyéné, 2002). La végétation est caractérisée d'une forêt dense semi-décidue (fortement soumise aux actions anthropiques).

Le climat est équatorial avec des précipitations moyennes interannuelles qui oscillent autour de $1562,7 \mathrm{~mm}$ et une température moyenne interannuelle de $23,93^{\circ} \mathrm{C}$ respectivement pour les périodes 1926 2018 et 1951-2018. Toutefois, ces précipitations moyennes interannuelles sont inférieures à la moyenne régionale qui oscille autour de $1600 \mathrm{~mm} / \mathrm{an}$ (Olivry, 1986 ; Suchel, 1987). La figure 2 fait ressortir alors quatre saisons à savoir : une grande saison pluvieuse (mi-septembre à mi-novembre) ; une grande saison sèche (mi-novembre à mi-mars) ; une petite saison pluvieuse (mi-mars à mi-juin) et une petite saison sèche (mi-juin à mi-septembre).

Les principales activités génératrices de revenue sont celles liées aux services administratifs, celles développées dans les Petites et Moyennes Entreprises (P.M.E) et les Petites et Moyennes Industries (P.M.I) pour les populations situées dans les quartiers à habitats modernes, évolutifs et plus ou moins confortables. Les populations des quartiers à habitats spontanés, situés dans les zones marécageuses ont des habitudes rurales avec pour activités principales l'agriculture et l'élevage.

\section{3. $\quad$ Matériels et Méthodes}

Cette étude est basée sur une approche méthodologique pluridisciplinaire qui consiste conjointement en (1) un suivi piézométrique et hydrométrique des ouvrages hydrauliques (puits et sources aménagés ou non préalablement choisis; (2) l'élaboration des cartes piézométriques ; (3) la réalisation des essais in situ de perméabilité et détermination du coefficient de perméabilité; (4) l'échantillonnage des sols pour les analyses granulométriques ; (5) l'échantillonnage et la conservation des eaux pour les analyses physico-chimiques et bactériologiques ; et enfin (6) l'établissement du bilan hydrique. 


\subsection{Données hydrométrique et piézométrique}

Cette étape a consisté à recenser préalablement à l'aide d'un fond de carte topographique et d'un récepteur GPS (Global Positioning System) les coordonnées géographiques (longitude, latitude et altitude) de tous les ouvrages hydrauliques existants (puits et sources aménagés ou non) dans le bassin versant de Nkié. A l'issu de cette investigation 136 ouvrages ont été recensés soient 128 puits et 08 sources. Compte tenu de l'implantation souvent onéreuse des piézomètres, 35 ouvrages soient 30 puits et 05 sources, ont fait l'objet d'un suivi piézométrique et hydrométrique respectivement (Figure 1).

Les critères de choix de ces ouvrages portent sur l'accessibilité, la représentativité, la répartition spatiale, la topographie, l'accord du propriétaire, le débit relativement élevé et la sollicitation pour des besoins de consommation par la population pour certaines sources.

Le suivi piézométrique s'est déroulé durant une année hydrologique allant de septembre 2017 à août 2018 à une cadence bimensuelle. Le niveau piézométrique $\mathrm{H}(\mathrm{m})$ a été déterminé suivant la formule dans le cas d'une nappe libre (Castany, 1998) et la profondeur d'eau (p) dans l'ouvrage a été déterminée à partir d'une sonde électrique lumineuse et sonore.

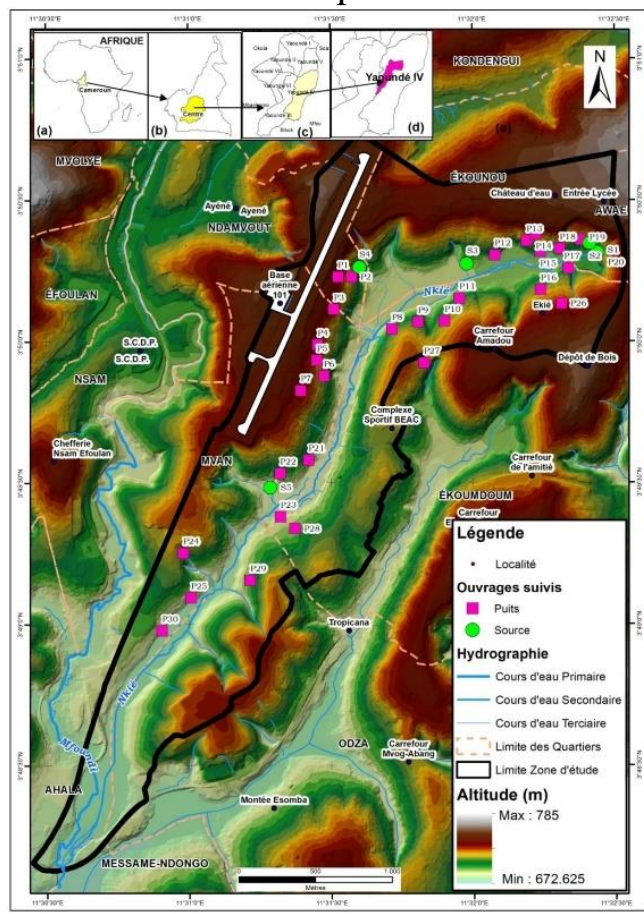

Figure 1. (a) Localisation du Cameroun en Afrique ; (b) Localisation de la Région du Centre dans le Cameroun ; (c) Localisation du Département d'étude ; (d) Localisation du bassin versant du Nkié dans l'Arrondissement d'étude ; (e) Localisation géographique, géomorphologie du bassin versant du Nkié ainsi que les ouvrages hydrauliques suivis (puits et source). 


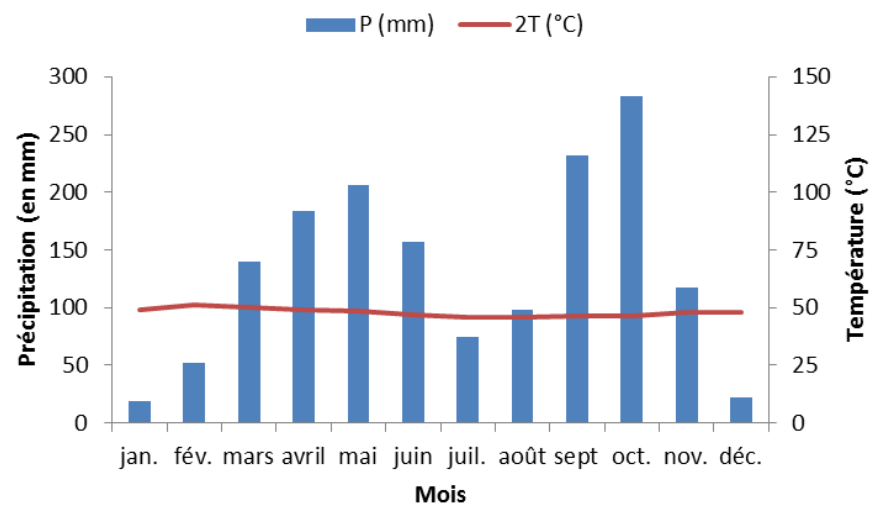

Figure 2. Diagramme ombrothermique de Bagnouls et Gaussen (1957) de la ville de Yaoundé, Période 1951-2018 (source : Station météorologique de la Base aérienne 101)

$$
H=Z-p
$$

Avec: $\mathrm{H}=$ Niveau piézométrique $(\mathrm{m}) ; \mathrm{Z}=$ altitude de la surface topographique $(\mathrm{en} \mathrm{m}) ; \mathrm{p}=$ profondeur du plan d'eau dans le piézomètre $(\mathrm{m})$ ou niveau statique.

Le suivi hydrométrique s'est également déroulé à la même période et à la même cadence que le suivi piézométrique. La méthode de jaugeage volumétrique a été utilisée. Elle consiste à noter le temps à partir d'un chronomètre que met une source d'eau pour remplir un seau de capacité connue. Le débit peut être calculé à partir de la formule suivante :

$$
Q=V / t
$$

Avec $\mathrm{Q}=$ débit d'écoulement $\left(1 . \mathrm{s}^{-1}\right) ; \mathrm{V}=$ volume du seau (l) $; \mathrm{t}=$ temps de remplissage (s).

\subsection{Caractérisation physique des sols}

Les essais in situ de perméabilité ont été effectués suivant la méthode de Porchet (1931) au niveau d'une toposéquence où 4 (quatre) horizons à savoir l'horizon meuble superficiel, l'horizon meuble nodulaire, l'horizon meuble rouge tacheté et l'allotérite peuvent être identifiés. Elle consiste à suivre, en fonction du temps, l'infiltration d'une quantité d'eau versée dans un trou de tarière de rayon connu. Des échantillons de sols remaniés de ces mêmes horizons ont par la suite été prélevés suivant le profil de sol, puis conservés dans des plastiques pour des analyses granulométriques au laboratoire de Géotechnique et Génie Civil de L'Ecole Nationale Supérieure Polytechnique de Yaoundé.

A partir des données des essais de perméabilité, lesdits coefficients ont été calculés suivant la formule (3) suivante.

$$
(2 K / 2,3 R) t=-\log (h+R / 2)+\text { cste }
$$


Avec $\mathrm{K}$ : coefficient de perméabilité $\left(\mathrm{en} \mathrm{m}_{\mathrm{s}} \mathrm{s}^{-1}\right)$; $\mathrm{R}$ : rayon du trou (en $\left.\mathrm{m}\right) ; \mathrm{h}$ : hauteur d'eau dans le trou (en $\mathrm{m}$ ) ; $\mathrm{t}$ : temps mis pour une infiltration.

Les analyses granulométriques ont également été effectuées sur ces différents échantillons. Les données issues de ces analyses ont permis de tracer les courbes granulométriques de chaque horizon. Le coefficient d'uniformité permettant de caractériser l'étalement de la granulométrie a ainsi été calculé suivant la formule (4).

$$
C u=d_{60} / d_{10}
$$

Avec $\mathrm{d}_{10}$ : Diamètre des grains correspondant à $10 \%$ de tamisât cumulé $: \mathrm{d}_{60}$ Diamètre des grains correspondant à $60 \%$ de tamisât cumulé.

La nature lithologique des différents horizons a été déterminée à partir du diagramme triangulaire de classification des produits d'altération d'Ekodeck(1984).

\subsection{Elaboration des cartes piézométriques}

L'élaboration des cartes piézométriques de l'aquifère à nappe libre du bassin versant du Nkié s'est faite par la méthode d'interpolation par krigeage. C'est une méthode géostatistique qui permet de réaliser l'interpolation approximative, spatiale d'une variable régionalisée par calcul de l'espérance mathématique d'une variable aléatoire, utilisant l'interprétation et la modélisation du variogramme expérimental. Le logiciel Surfer 14 a été utilisé à cet effet. L'établissement de la carte piézométrique a pour but de déterminer les principales directions de l'écoulement souterrain et les aires d'alimentation de la nappe. Ces cartes schématisent d'une part les fonctions capacitives et conductrices des réservoirs, et d'autre part le comportement hydrodynamique de l'aquifère. Les cartes piézométriques minimale, maximale et moyenne annuelle ont ainsi été élaborées correspondant respectivement au mois de l'année ayant les niveaux piézométriques minimale, maximale et du niveau piézométrique moyen de chaque ouvrage hydraulique durant l'année d'étude. Une carte de fluctuation de l'aquifère a été établie par différence des niveaux piézométriques maximale et minimale.

\subsection{Prélèvements et analyses des échantillons eaux}

La source la plus sollicitée par les populations et ayant le débit le plus important, a été identifiée puis prélevée à une cadence saisonnière en vue des analyses physicochimiques et bactériologiques. Toutefois, les paramètres tels que la température, la conductivité et le $\mathrm{pH}$ ont été fait in situ. Les analyses physico-chimiques des cations majeurs $\left(\mathrm{Na}^{+}, \mathrm{Ca}^{2+}, \mathrm{K}^{+}, \mathrm{Mg}^{+}\right)$, anions majeurs $\left(\mathrm{Cl}^{-}, \mathrm{NO}_{3}{ }^{-}, \mathrm{HCO}_{3}{ }^{-}, \mathrm{SO}_{4}{ }^{2-}\right)$ et quelques éléments en trace (Fer, Silice) ont été faite au Laboratoire d'Analyse Géochimique des Eaux (LAGE) de l'Institut de Recherche Géologiques et Minières du Ministère de la Recherche Scientifique et de l'Innovation à Yaoundé-Cameroun par la méthode de 
chromatographie ionique à phase liquide. Le logiciel Diagrammes de Roland SMILLER du Laboratoire d'Hydrogéologie d'Avignon a permis par la suite de traiter les données obtenues.

L'analyse bactériologique de l'échantillon de la source ayant préalablement subi les analyses physicochimiques de la saison sèche a été effectuée au Laboratoire Waste Water Research Unit de l'Université de Yaoundé ICameroun. La méthode utilisée est « la colorimétrie sur membrane filtrante » (Turgeonet al., 2011 ;Apha 2012; Akronget al., 2019). Elle permet d'identifier les bio-indicateurs de contamination fécale que Rodier(2009) définit comme des bactéries d'habitat fécal normal et exclusif. Il s'agit notamment des streptocoques fécaux (SF) et coliformes fécaux (CF).

$$
\mathrm{UFC}=\frac{\text { Nombre de colonies comptées }}{\text { Volume d'échantillon filtré }(\mathrm{ml})} \times 100 \mathrm{ml}
$$

Avec UFC $=$ Unité Formant Colonies pour $100 \mathrm{ml}$.

\subsection{Bilan Hydrique}

Le bilan hydrique a été établi par la méthode de Thornthwaite(1954). Cette méthode permet d'évaluer le déficit d'écoulement mensuel et tient compte de la réserve du sol d'un mois à l'autre (Remenieras, 1972). Elle consiste à calculer tout d'abord pour chaque mois, une évapotranspiration potentielle (ETP), que l'on peut assimiler au pouvoir évaporant de l'atmosphère. Cette évaporation potentielle est fonction de la température moyenne mensuelle par rapport aux indices thermiques mensuels (i), calculés à partir de l'équation 6 suivante et de la durée astronomique du jour.

$$
i=(t / 5)^{1,514} \text {. }
$$

Par la suite, on peut calculer l'ETP mensuelle à partir de l'équation 7 suivante.

$$
\begin{gathered}
\operatorname{ETP}_{i}=16\left(\frac{10 t_{i}}{I}\right)^{\propto}(7) \\
\text { Avec : } \\
\propto=1,6\left(\frac{I}{100}\right)+0,5 \quad \text { et } I=\sum_{i=1}^{12} i_{i}
\end{gathered}
$$

Avec $\mathrm{T}$ : température moyenne mensuelle ; I : indice thermique annuel ; i : indice thermique mensuel.

L'évapotranspiration potentielle calculée sera ainsi confrontée aux précipitations afin de déterminer l'évapotranspiration réelle (ETR), la réserve facilement utilisable (RFU), la variation de la réserve facilement utilisable ( $\triangle \mathrm{RFU}$ ), le water surplus (WS), l'écoulement total (S), l'écoulement de surface (QS), l'écoulement souterrain (QW), le déficit agricole (DA) et le coefficient mensuel d'humidité $(\alpha)$. L'infiltration a été calculée à partir de l'équation (8).

$$
I=P-\left(E T R+H_{e}\right)(8)
$$


Avec I : infiltration moyenne interannuelle (en mm) ; ETR : évapotranspiration réelle interannuelle (en $\mathrm{mm})$; $\mathrm{P}$ : précipitation moyenne interannuelle; He : lame d'eau écoulée moyenne interannuelle.

La lame d'eau écoulée moyenne interannuelle a été déduite de la relation traduite par l'équation 9 suivante proposée par Olivry(1986).

$$
H e=0,973 P-1047 \text { (9) }
$$

Avec $\mathrm{H}_{\mathrm{e}}$ : lame d'eau écoulée moyenne interannuelle $(\mathrm{mm}) ; \mathrm{P}$ : précipitation moyenne interannuelle $(\mathrm{mm})$.

Cette relation semble être vérifiée pour les régions à précipitations supérieures à $1500 \mathrm{~mm}$ du bassin de la Sanaga, du climat tropical de transition et des bassins côtiers (Sighomnou, 2004).

\section{Résultats et Discussions}

\subsection{Caractérisation physique des sols}

Les résultats d'analyse granulométrique et de lithologie ont permis de mieux appréhender les caractéristiques physiques des altérites du bassin versant du Nkié. La distribution granulométrique des produits d'altération (Tableau 1) révèle que chaque horizon est formé des grains de diamètres variés. De même, le coefficient d'uniformité (CU), calculé pour chaque horizon est supérieur à 2,5 et les fractions fine et grenue des différents horizons sont respectivement comprises entre $24,69 \%$ et $95,31 \%$; et entre $4,69 \%$ et $75,31 \%$ (Tableau 2) illustrant ainsi une granulométrie variée. Les teneurs en particules fines et grenues sont respectivement plus importantes dans l'horizon superficiel et dans le nodulaire. Il existe donc d'une manière globale, une diminution de la taille des particules du sol de la profondeur vers la surface du profil d'altération. Cependant, il faut noter une perturbation au niveau de l'horizon nodulaire (horizon médian) qui comporte plus de particules grenues que les horizons sous-jacents. Cette perturbation granulométrique proviendrait du fait que le niveau nodulaire est soumis à un processus d'aggradation par accumulation d'oxy-hydroxydes de fer qui génère des matériaux grossiers de l'ordre du centimètre (Onana, 2001). Les niveaux supérieur et inférieur subissent le processus inverse respectivement le soutirage et la dégradation par fragmentation.

Tableau 1. Distribution granulométrique des produits d'altération du bassin versant du Nkié

\begin{tabular}{ccccccc}
\hline $\begin{array}{c}\text { Formations } \\
\text { d'altération }\end{array}$ & Cailloux & Graviers & $\begin{array}{c}\text { Sables } \\
\text { grossiers }\end{array}$ & $\begin{array}{c}\text { Sables } \\
\text { fins }\end{array}$ & $\begin{array}{c}\text { Argiles+ } \\
\text { Limons }\end{array}$ & Totaux \\
\hline Horizonsuperficiel & 0,00 & 0,00 & 4,69 & 38,42 & 56,89 & 100 \\
& & & & & & \\
& 13,39 & 59,63 & 2,29 & 17,80 & 6,89 & 100 \\
Horizon nodulaire & 0,00 & 2,74 & 10,11 & 70,26 & 16,89 & 100 \\
Horizon tacheté & 2,43 & 28,26 & 21,40 & 29,01 & 18,9 & 100 \\
$\quad$ Allotérite & & & & &
\end{tabular}


Tableau 2. Paramètres granulométriques des produits d'altération du bassin versant du Nkié

\begin{tabular}{ccccc}
\hline & Horizonsuperficiel & $\begin{array}{c}\text { Horizon } \\
\text { nodulaire }\end{array}$ & $\begin{array}{c}\text { Horizon } \\
\text { tacheté }\end{array}$ & Allotérite \\
\hline $\begin{array}{c}\text { Particules } \\
\text { grenues } \\
(\phi>0,2 \mathrm{~mm})\end{array}$ & 4,69 & 75,31 & 12,85 & 52,09 \\
$\begin{array}{c}\text { Particules } \\
\text { fines }\end{array}$ & 95,31 & 24,69 & 87,15 & 47,91 \\
$\begin{array}{c}\phi<0,2 \mathrm{~mm}) \\
\text { Diamètre d } \\
\text { Diamètre }\end{array}$ & 0,017 & 10 & 0,038 & 0,63 \\
$\begin{array}{c}\text { efficace }\left(\mathrm{d}_{10}\right) \\
\text { Coefficient }\end{array}$ & 0,002 & 0,075 & 0,01 & 0,004 \\
$\begin{array}{c}\text { d'uniformité } \\
(\mathrm{U})\end{array}$ & 8,5 & 133,33 & 3,8 & 157,5 \\
\hline
\end{tabular}

Le digramme triangulaire de classification d'Ekodeck(1984) illustre que les différents produits d'altération du bassin versant du Nkié sont globalement sableux (Figure3). Ce diagramme permet de dire que l'allotérite est un sable graveleux, l'horizon rouge tacheté correspond à un sable, l'horizon nodulaire est un gravier sableux et l'horizon superficiel meuble équivaut à une argile sableuse. Ces formations lithologiques sont réparties en deux zones, à savoir la zone saturée et la zone non-saturée. Le sable graveleux représente la zone saturée qui est caractérisée par le complexe solide/eau. La zone non-saturée est constituée ici de bas en haut d'un sable, un gravier sableux et une argile sableuse. C'est le domaine de transport d'eau et d'aération du milieu.

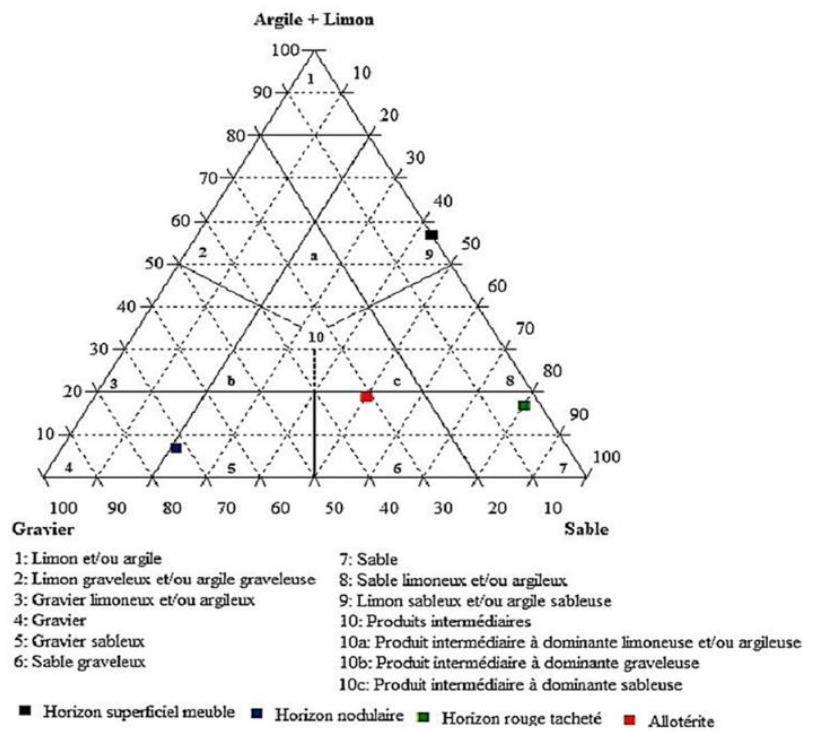

Figure 3. Représentation des produits d'altération du bassin versant du Nkié dans le diagramme triangulaire de classification d'Ekodeck(1984) 
D'une manière générale, les valeurs de perméabilité des différents produits d'altération du bassin versant du Nkié varient entre $1,8 \cdot 10^{-6} \mathrm{~m} \cdot \mathrm{s}^{-1}$ et $5,92 \cdot 10^{-5}$ $\mathrm{m} . \mathrm{s}^{-1}$ et diminuent de la profondeur vers la surface du profil (Tableau 3). Ces valeurs illustrent ainsi que les différents horizons sont peu perméables à assez perméables (Collin, 2004). La perméabilité est alors fonction de la granulométrie et du degré d'interconnections entre les vides d'un réservoir (Castany, 1998). Un niveau riche en particules grenues sera d'autant plus perméable qu'un milieu riche en particules argileuses. Cependant, les argiles sableuses superficielles sont les plus perméables malgré leur enrichissement en particules argileuses qui ont une forte capacité de rétention. Cet ordre de perméabilité serait donc influencé par la présence des macrospores causés par l'action des racines des plantes ou des organismes vivants (vers de terre, insectes), des fentes de dessiccation souvent présentes dans les matériaux argileux (Parriauxet al., 2002). Les valeurs de perméabilité ainsi obtenues dans le bassin versant du Nkié sont du même ordre de grandeur que celles trouvées par: Bon (2008) dans le bassin de l'Olezoa.

Tableau 3. Valeurs de perméabilité des formations lithologiques du Bassin versant de Nkié

\begin{tabular}{|c|c|c|c|c|}
\hline Horizon & $\begin{array}{l}\text { Horizon } \\
\text { superficiel } \\
\text { meuble }\end{array}$ & $\begin{array}{l}\text { Horizon } \\
\text { Nodulaire }\end{array}$ & $\begin{array}{c}\text { Horizon } \\
\text { Rouge } \\
\text { Tacheté }\end{array}$ & Allotérite \\
\hline $\begin{array}{l}\text { Formation } \\
\text { lithologique }\end{array}$ & Argile sableuse & $\begin{array}{l}\text { Gravier } \\
\text { sableux }\end{array}$ & Sable & $\begin{array}{c}\text { Sable } \\
\text { Graveleux }\end{array}$ \\
\hline Perméabilité (m.s $\left.{ }^{-1}\right)$ & $5,92 \cdot 10^{-5}$ & $1,80.10^{-6}$ & $7,43.10^{-6}$ & $1,09 \cdot 10^{-5}$ \\
\hline
\end{tabular}

\subsection{Caractérisation hydrométrique des sources d'eau}

Les débits journaliers des sources obtenus varient entre $0,061 . \mathrm{s}^{-1}$ $\left(0,22 \mathrm{~m}^{3} \cdot \mathrm{h}^{-1}\right)$ et $0,691 . \mathrm{s}^{-1}\left(2,5 \mathrm{~m}^{3} \cdot \mathrm{h}^{-1}\right)$ en saison sèche et entre $0,081 . \mathrm{s}^{-1}(0,3$ $\left.\mathrm{m}^{3} \cdot \mathrm{h}^{-1}\right)$ et $0,861 \cdot \mathrm{s}^{-1}\left(3,1 \mathrm{~m}^{3} \cdot \mathrm{h}^{-1}\right)$ en saison pluvieuse.

Le débit moyen annuel correspondant est de $0,231 . \mathrm{s}^{-1}$ soit $0,83 \mathrm{~m}^{3} \cdot \mathrm{h}^{-1}$. Les débits moyens mensuels des sources sont compris entre $0,161 . \mathrm{s}^{-1}(0,58$ $\left.\mathrm{m}^{3} \cdot \mathrm{h}^{-1}\right)$ et $0,281 \cdot \mathrm{s}^{-1}\left(1,01 \mathrm{~m}^{3} \cdot \mathrm{h}^{-1}\right)$. Les débits journaliers se rapprochent de ceux trouvés par : Ntep (2005) dans le bassin versant de la Mingoa $(0,22$ à $\left.3,06 \mathrm{~m}^{3} \cdot \mathrm{h}^{-1}\right)$; Feumba(2005) dans le bassin versant d'Ekozoa $(0,17$ à 3,18 $\left.\mathrm{m}^{3} \cdot \mathrm{h}^{-1}\right)$ et Kalla(2007) dans le bassin versant du Ntem $\left(0,36\right.$ à $\left.3,14 \mathrm{~m}^{3} \cdot \mathrm{h}^{-1}\right)$. La Figure 4 présente l'hydrogramme moyen mensuel des débits des sources du bassin versant du Nkié, en fonction des précipitations. On note cependant que les variations de ces débits suivent celles des précipitations dans la zone. Toutefois, il existe un décalage entre les mises en charge de la nappe par rapport aux précipitations. Ce décalage témoigne d'une faible vitesse d'infiltration qui serait liée aux épaisses couches de sol, à l'état fissural et aux axes de drainage à direction variée. Une corrélation établie entre les débits moyens annuels des sources et les altitudes (Figure 5) révèle que les 
débits des sources ne sont pas liés à l'altitude du point de résurgence (Ekodecket al., 2002). Les variations spatio-temporelles des débits des sources seraient donc en partie influencées par des fractures de socle oblitérées par les minéraux argileux. Les sources de plus grands débits (S3) seraient donc situées sur les principaux axes de drainage correspondant aux axes principaux de fracturation (N10-30, N40-50, N80-100, N110-120) (Mpesse, 1999 ; Bosso, 2000).

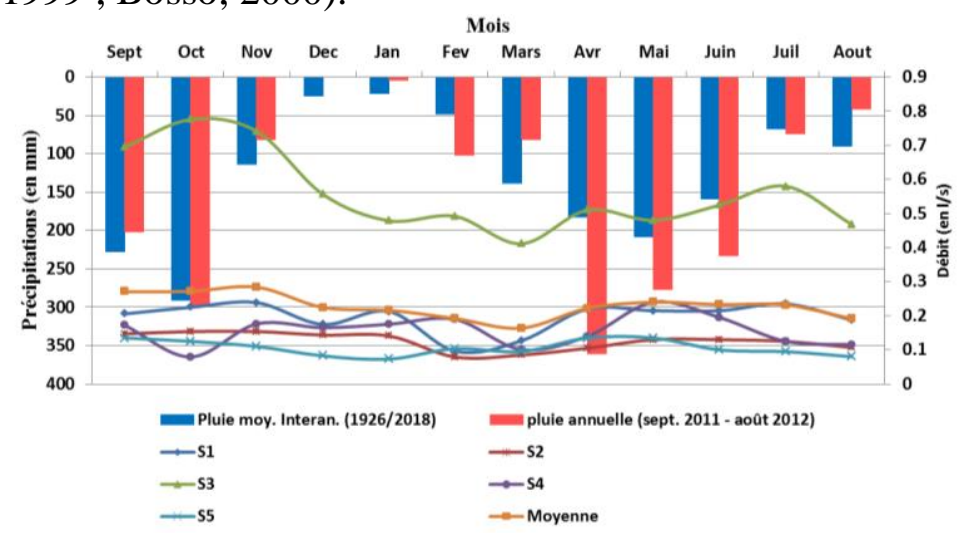

Figure 4. Hydrogramme moyen mensuel des débits des sources du bassin versant du Nkié en en fonction des précipitations.

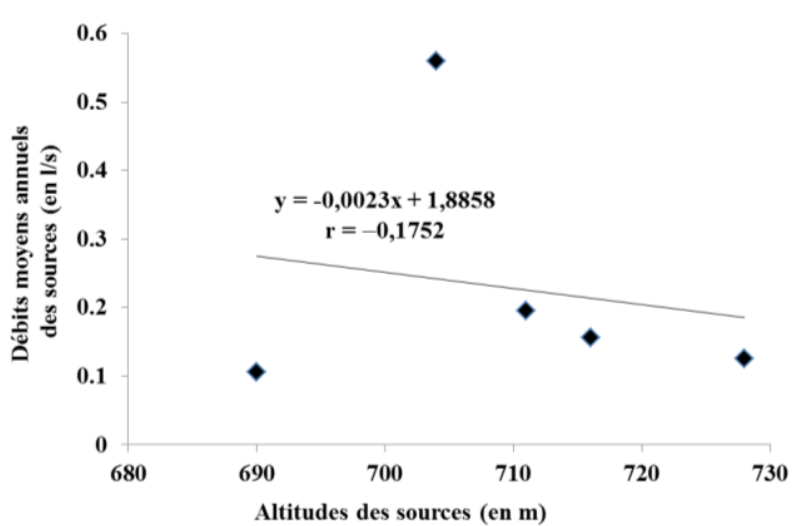

Figure 5. Droite régressive de la corrélation entre les altitudes et les moyennes annuelles des débits des sources

\subsection{Caractérisation piézométrique}

Les niveaux piézométriques varient entre 733,92 et $682,68 \mathrm{~m}$ pendant la période maximale, et entre 732,29 et 682,57 en période minimale. Les fluctuations piézométriques varient ainsi entre $0,1 \mathrm{~m}$ (zone de bas-fond) et 7,48 m (pour P3 situé au sommet des versants), soit une fluctuation moyenne annuelle de 1,36 m (Tableau 4). Cette fluctuation est relativement supérieure à celles trouvées dans d'autres bassins hydrographiques du Mfoundi comme 
ceux de l'Ekozoa (Feumba, 2005) soit 0,55 à 1,65m. ; de Ntem (Kalla, 2007) soit 0,1 à $1,91 \mathrm{~m}$; de l'Ebogo-Ewé (Priso, 2007) soit 0,08 à $0,88 \mathrm{~m}$

Tableau 4. Synthèse des données piézométriques du bassin versant du Nkié

\begin{tabular}{|c|c|c|c|c|}
\hline Ouvrages & NPMA (en m) & NPC (en m) & NPE (en m) & VNP (en m) \\
\hline $\mathrm{P}_{1}$ & 722,55 & 724,11 & 721,36 & 2,75 \\
\hline $\mathrm{P}_{2}$ & 704,70 & 705,78 & 704,13 & 1,65 \\
\hline $\mathrm{P}_{3}$ & 717,71 & 720,74 & 713,26 & 7,48 \\
\hline $\mathrm{P}_{4}$ & 715,21 & 717,25 & 714,4 & 2,85 \\
\hline $\mathrm{P}_{5}$ & 719,38 & 720,87 & 718,4 & 2,47 \\
\hline $\mathrm{P}_{6}$ & 705,21 & 705,5 & 704,77 & 0,73 \\
\hline $\mathrm{P}_{7}$ & 719,87 & 722,08 & 718,64 & 3,44 \\
\hline $\mathrm{P}_{8}$ & 685,50 & 686,04 & 685,2 & 0,84 \\
\hline $\mathrm{P}_{9}$ & 683,38 & 684,07 & 682,57 & 1,5 \\
\hline $\mathrm{P}_{10}$ & 689,74 & 690,47 & 689,1 & 1,37 \\
\hline $\mathrm{P}_{11}$ & 686,39 & 687,28 & 685,72 & 1,56 \\
\hline $\mathrm{P}_{12}$ & 708,44 & 708,79 & 708,39 & 0,4 \\
\hline $\mathrm{P}_{13}$ & 721,77 & 721,74 & 721,17 & 0,57 \\
\hline $\mathrm{P}_{14}$ & 733,05 & 733,92 & 732,29 & 1,63 \\
\hline $\mathrm{P}_{15}$ & 721,17 & 721,59 & 721,49 & 0,1 \\
\hline $\mathrm{P}_{16}$ & 699,49 & 699,78 & 698,36 & 1,42 \\
\hline $\mathrm{P}_{17}$ & 699,71 & 700,25 & 700,04 & 0,21 \\
\hline $\mathrm{P}_{18}$ & 724,80 & 724,94 & 724,8 & 0,14 \\
\hline $\mathrm{P}_{19}$ & 728,51 & 728,35 & 728,04 & 0,31 \\
\hline $\mathrm{P}_{20}$ & 716,15 & 716,44 & 715,64 & 0,8 \\
\hline $\mathrm{P}_{21}$ & 687,17 & 687,5 & 687,23 & 0,27 \\
\hline $\mathrm{P}_{22}$ & 688,07 & 688,22 & 688,03 & 0,19 \\
\hline $\mathrm{P}_{23}$ & 687,90 & 688,22 & 688,03 & 0,19 \\
\hline $\mathrm{P}_{24}$ & 701,19 & 701,5 & 700,4 & 1,1 \\
\hline $\mathrm{P}_{25}$ & 682,83 & 683,17 & 682,69 & 0,48 \\
\hline $\mathrm{P}_{26}$ & 710,29 & 711,64 & 709,28 & 2,36 \\
\hline $\mathrm{P}_{27}$ & 702,58 & 702,88 & 701,99 & 0,89 \\
\hline $\mathrm{P}_{28}$ & 688,41 & 689,34 & 687,78 & 1,56 \\
\hline $\mathrm{P}_{29}$ & 694,35 & 694,42 & 694,16 & 0,26 \\
\hline $\mathrm{P}_{30}$ & 681,63 & 682,68 & 680,78 & 1,9 \\
\hline Моу. & 705,99 & 704,98 & 703,60 & 1,36 \\
\hline Méd. & 705,21 & 705,78 & 704,77 & 0,84 \\
\hline Max. & 733,05 & 733,92 & 732,29 & 7,48 \\
\hline Min. & 682,83 & 683,17 & 682,57 & 0,1 \\
\hline ET. & 16,17 & 16,38 & 15,96 & 1,58 \\
\hline CV. & 0,023 & 0,023 & 0,023 & 1,16 \\
\hline
\end{tabular}

NPMA : Niveaux piézométriques moyens annuels ; NPC : Niveaux piézométriques de crue ; NPE : Niveaux piézométriques d'étiage; VNP : Variations des niveaux piézométriques; Moy. Moyenne ; Méd. : Médiane ; Max. Maximum ; Min. : Minimum ; ET. Ecart-Type ; CV. Coefficient de Variation.

L'évolution des niveaux piézométriques mensuels en fonction des pluies montre que le mois de novembre représente la période des hautes eaux de l'aquifère à nappe libre du bassin versant du Nkié et mars celle des basses eaux (Figure 6). Les cartes piézométriques de l'aquifère à nappe libre du 
bassin versant du Nkié montre un écoulement non uniforme des eaux souterraines. Cela est caractérisé sur les cartes par une direction variée des lignes de courant qui sont soit divergentes soit convergentes (Figures 7 et 8). L'analyse des cartes piézométriques porte sur l'orientation des lignes de courant et la courbure des arcs des cercles. Ces cartes révèlent ainsi des zones à arc de cercle concentrique portant des lignes de courant convergentes et celles à arc de cercle concentrique et portant des lignes de courant divergentes. Ces zones constituent respectivement des zones de stockage et d'alimentation de l'aquifère à nappe libre du bassin versant du Nkié. Il faut noter que les zones de stockage de l'aquifère constituent des zones propices à l'implantation des ouvrages hydrauliques.

D'une manière générale les lignes de courant convergent vers le cours d'eau Nkié qui constitue à cet effet le principal axe de drainage de l'aquifère à nappe libre du bassin versant du Nkié. Ce cours d'eau peut donc être alimenté par la nappe en période d'étiage.

La différence d'altitude entre les données piézométriques maximale (05 novembre 2017) et minimale (24 mars 2018) a permis de tracer la carte de fluctuation annuelle de la surface piézométrique (Figure 8b). Cette dernière montre que les puits localisés sur les flancs de collines ont des fluctuations plus importantes que ceux situés dans les bas-fonds près du lit du cours d'eau Nkié. Cela s'expliquerait par la présence d'une matrice argileuse présente dans les bas-fonds qui réduit par ses propriétés de rétention et d'imperméabilité l'infiltration efficace. Cette zone est caractérisée par l'occupation pérenne de la nappe à faible profondeur.

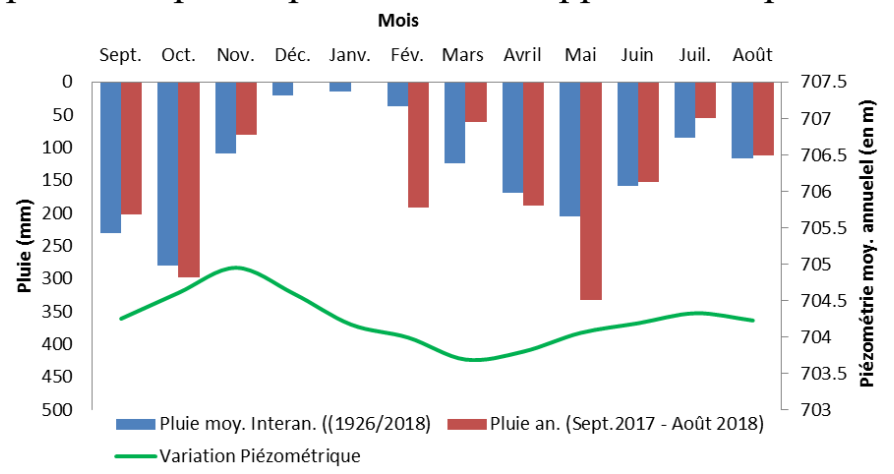

Figure 6. Relation pluie - niveaux piézométriques mensuels du bassin versant du Nkié 


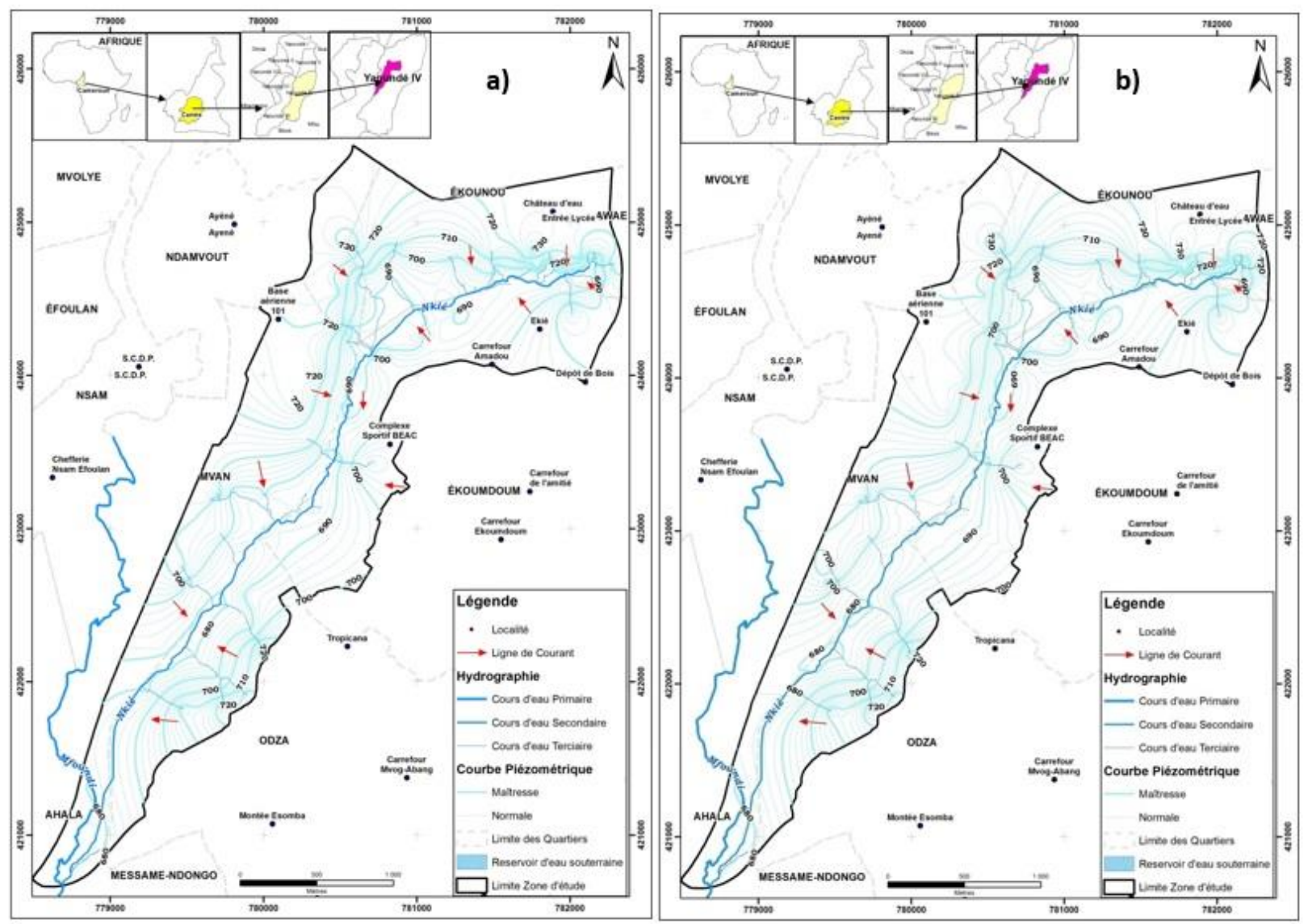

Figure 7. (a) Carte piézométrique maximale (b) Carte piézométrique minimale 


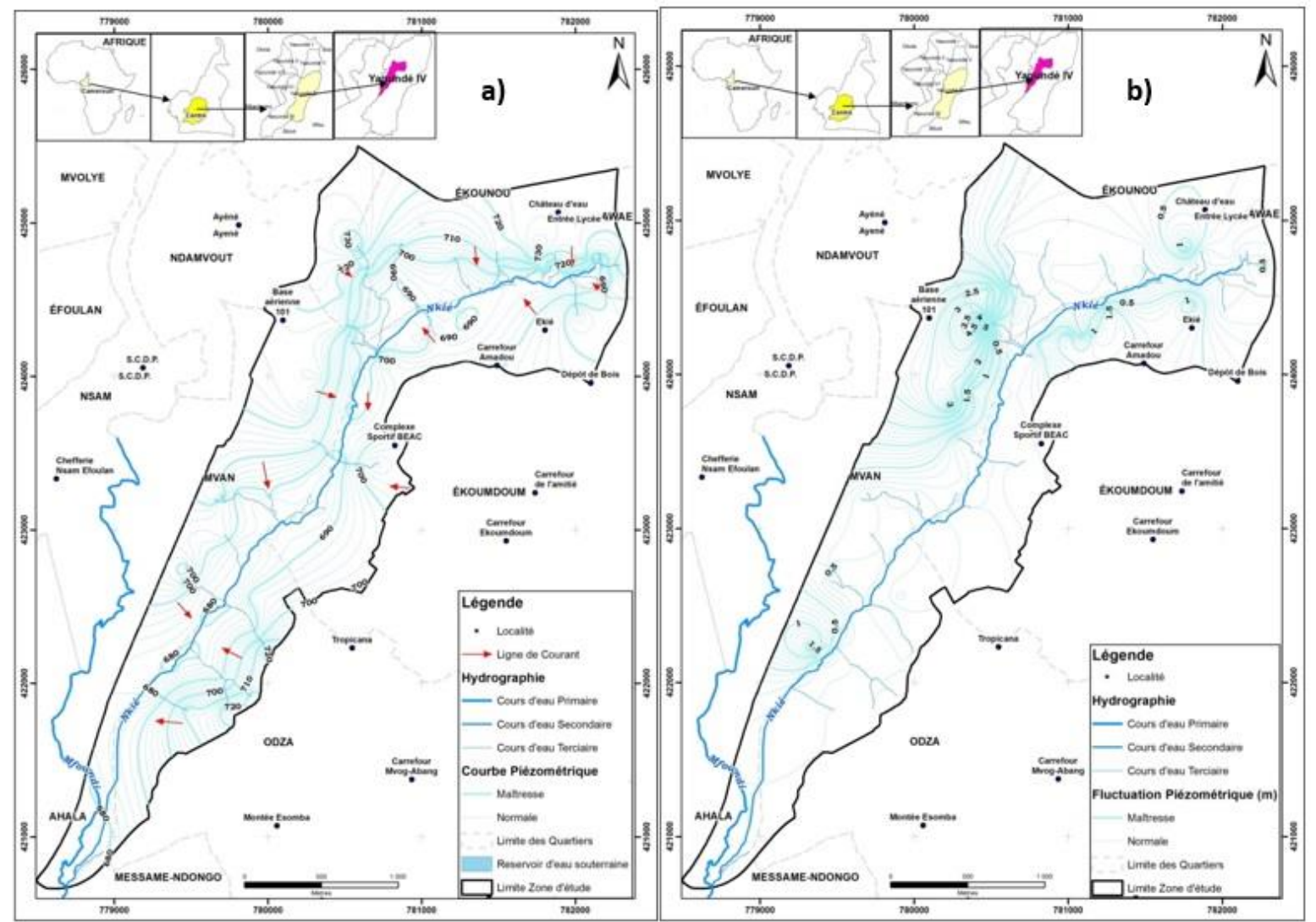

Figure 8. (a) Carte piézométrique moyenne annuelle (b) Carte de fluctuation annuelle de l'aquifère du bassin versant du Nkié 


\subsection{Bilan Hydrique}

Les paramètres du bilan hydrique ont été calculés pour la période allant de 1951 à 2018 (Tableau 5). Il en ressort que la précipitation moyenne interannuelle $(\mathrm{P})$, l'évapotranspiration réelle (ETR) et la lame d'eau écoulée (He) sont respectivement de $1584,52 \mathrm{~mm}, 991,71 \mathrm{~mm}$ et 494,74 mm. Par conséquent, l'infiltration sera de $98,07 \mathrm{~mm}$ soit un coefficient d'infiltration de $6,19 \%$. Les pertes d'eau sont donc estimées $93,81 \%$ soit $62,59 \%$ par évapotranspiration et $31,22 \%$ par écoulement. Le coefficient d'infiltration ainsi calculé se rapproche de celui obtenu par Kenzo (2008) soit 6,1\% dans le bassin hydrographique de Ntsomo. Il est par contre supérieur à ceux obtenus par Ntep (2005) soit 5,57\%, Kuate (2008) soit 5,1\% respectivement dans les bassins versant de la Mingoa et de la Biyemé, par Fouepe (2011) soit 5,7\% dans le bassin versant de l'Anga'a par la méthode hybride de fluctuation de l'eau. L'évolution des paramètres ainsi obtenus (Figure 9) montre que la période d'excédent pluviométrique va de mi-mars à novembre traduisant la recharge de l'aquifère à nappe libre tandis que celle du déficit pluviométrique va de décembre à mi-mars et correspond à la vidange de l'aquifère à nappe libre. La période de déficit pluviométrique est caractérisée par une baisse progressive des débits des sources et des niveaux piézométriques. Cette conclusion est en accord avec celle de (Djeudaet al., 1999). D'après cet auteur, la recharge de l'aquifère à nappe libre se fait d'avril à novembre et la vidange de décembre à mars.

Tableau 5. Paramètres du bilan hydrique, calculés selon la méthode de Thornthwaite (1954)

\begin{tabular}{|c|c|c|c|c|c|c|c|c|c|c|c|c|c|}
\hline & M & $\mathbf{A}$ & M & $\mathbf{J}$ & $\mathbf{J}$ & $\mathbf{A}$ & $\mathbf{S}$ & $\mathbf{O}$ & $\mathbf{N}$ & D & $\mathbf{J}$ & $\mathbf{F}$ & Totaux \\
\hline $\mathrm{P}(\mathrm{mm})$ & 139,73 & 183,79 & 206,29 & 156,45 & 74,27 & 97,82 & 232,13 & 283,55 & 117,38 & 22,41 & 18,82 & 51,90 & 1584,52 \\
\hline $\mathrm{ETP}(\mathrm{mm})$ & 106,40 & 100,71 & 97,59 & 88,64 & 81,25 & 81,61 & 85,77 & 83,98 & 92,84 & 93,42 & 100,12 & 111,58 & 1123,92 \\
\hline ETR (mm) & 94,04 & 87,91 & 97,59 & 88,64 & 74,56 & 81,61 & 85,77 & 83,98 & 92,84 & 93,42 & 59,45 & 51,90 & 991,71 \\
\hline RFU (mm) & 44,90 & 100,00 & 100,00 & 100,00 & 100,00 & 100,00 & 100,00 & 100,00 & 100,00 & 40,63 & 0,00 & 0,00 & 885,53 \\
\hline DRFU (mm) & 44,90 & 55,10 & 0,00 & 0,00 & 0,00 & 0,00 & 0,00 & 0,00 & 0,00 & $-59,37$ & $-40,63$ & 0,00 & 0,00 \\
\hline $\mathrm{WS}(\mathrm{mm})$ & 0,00 & 40,78 & 108,70 & 67,81 & 0,00 & 16,21 & 146,35 & 199,56 & 24,54 & 0,00 & 0,00 & 0,00 & 603,95 \\
\hline $\mathrm{S}(\mathrm{mm})$ & 0,00 & 39,02 & 128,21 & 131,92 & 65,96 & 49,19 & 170,95 & 285,03 & 167,05 & 83,53 & 41,76 & 20,88 & 1183,50 \\
\hline $\mathrm{QS}(\mathrm{mm})$ & 0,00 & 19,51 & 64,10 & 65,96 & 32,98 & 24,59 & 85,47 & 142,52 & 83,53 & 41,76 & 20,88 & 10,44 & 591,75 \\
\hline QW (mm) & 0,00 & 19,51 & 64,10 & 65,96 & 32,98 & 24,59 & 85,47 & 142,52 & 83,53 & 41,76 & 20,88 & 10,44 & 591,75 \\
\hline $\mathrm{DA}(\mathrm{mm})$ & 0,00 & 0,00 & 0,00 & 0,00 & 0,00 & 0,00 & 0,00 & 0,00 & 0,00 & 0,00 & 40,67 & 59,68 & 100,35 \\
\hline P-ETP (mm) & 33,33 & 83,08 & 108,70 & 67,81 & $-6,98$ & 16,21 & 146,35 & 199,56 & 24,54 & $-71,01$ & $-81,30$ & $-59,68$ & 460,60 \\
\hline a $(\%)$ & 0,31 & 0,82 & 1,11 & 0,77 & $-0,09$ & 0,20 & 1,71 & 2,38 & 0,26 & $-0,76$ & $-0,81$ & $-0,53$ & \\
\hline
\end{tabular}

$\mathrm{P}$ : précipitations ; ETP : évapotranspiration potentielle ; ETR : évapotranspiration réelle ; $\mathrm{RFU}$ : réserve facilement utilisable ; $\triangle \mathrm{RFU}$ : variation de la réserve facilement utilisable ; WS : water surplus QS : lame d'eau disponible pour l'écoulement de surface ; DA : déficit agricole $\mathrm{S}$ : lame d'eau disponible pour l'écoulement souterrain ; $\alpha$ : coefficient mensuel d'humidité. 


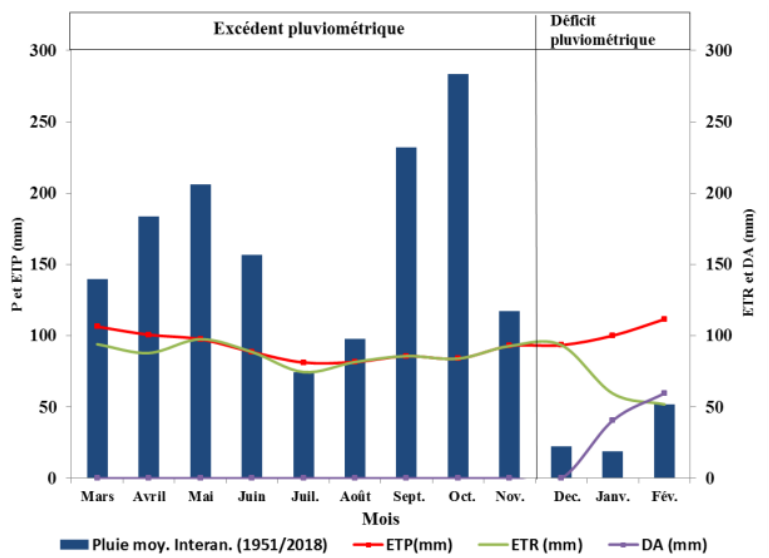

Figure 9. Evolution de quelques paramètres du bilan hydrique établi selon la méthode de Thornthwaite(1954).

\subsection{Caractéristiques physico-chimique et bactériologique des eaux}

Les cations majeurs représentés par le sodium $(\mathrm{Na}+)$, potassium $(\mathrm{K}+)$, magnésium $(\mathrm{Mg} 2+)$ et calcium $(\mathrm{Ca} 2+)$ ont des concentrations respectives de $(354,35 \mu \mathrm{eq} / \mathrm{l}), 5,56 \mu \mathrm{eq} / \mathrm{l}, 59,08 \mu \mathrm{eq} / \mathrm{l}, 130,04 \mu \mathrm{eq} / \mathrm{l}$ et 97 $\mu$ eq/l durant la saison pluvieuse et de 1653,91 $\mu \mathrm{eq} / \mathrm{l}, 61,38 \mu \mathrm{eq} / \mathrm{l}, 125,93$ $\mu \mathrm{eq} / 1$ et $87,5 \mu \mathrm{eq} / 1$ en saison sèche (Tableau 6). Ces cations sont essentiellement produits au cours de l'hydrolyse des minéraux primaires suivant l'équation :

Minéraux primaires $+\mathrm{CO} 2+\mathrm{H} 2 \mathrm{O} \longrightarrow \mathrm{HCO} 3-+$ minéraux secondaires + cations + silice (I). (Garrels, 1976).

A cet effet, les ions $\mathrm{Mg} 2+$ et $\mathrm{Ca} 2+$ proviennent de la dissolution des éléments ferromagnésiens et de l'altération des plagioclases calciques (Hem, 1985 ; Ameen, 2019) respectivement contenus dans le gneiss. Les ions $\mathrm{Na}+$ et $\mathrm{K}+$ quant à eux proviennent de l'altération et du lessivage de la roche pour le sodium (Zianiet al., 2017) en particulier et l'utilisation des pesticides pour le potassium (Melo et al., 2012). L'eau souterraine au cours de son séjour et de son écoulement dans les formations hydrogéologiques perméables subit des échanges géochimiques avec son réservoir permettant de modifier les caractéristiques de l'eau (Castany, 1982). La TZ+ donne 640,47 $\mu$ éq/l en saison pluvieuse et 1928,72 $\mu$ éq/l en saison sèche (Tableau 6). La forte valeur de $\mathrm{TZ}^{+}$enregistrée en saison sèche est liée à l'évaporation et aux rejets anthropiques diverses. Les anions majeurs représentées par le chlorure $\left(\mathrm{Cl}^{-}\right)$, nitrate $\left(\mathrm{NO}_{3}{ }^{-}\right)$, sulfate $\left(\mathrm{SO}_{4}{ }^{2-}\right)$, bicarbonate $\left(\mathrm{HCO}_{3}{ }^{-}\right)$ont des concentrations respectives de $626,76 \mu \mathrm{eq} / 1,1505,97 \mu \mathrm{eq} / \mathrm{l}, 6,25 \mu \mathrm{eq} / 1$ et $30 \mu \mathrm{eq} / \mathrm{l}$ en saison pluvieuse et de $1669 \mu \mathrm{eq} / 1,58 \mu \mathrm{eq} / 1,1492,42 \mu \mathrm{eq} / 1,36,25 \mu \mathrm{eq} / \mathrm{l}$ et $0 \mu \mathrm{eq} / \mathrm{l}$ en saison sèche (Tableau 6). La TZ ${ }^{-}$calculée est de 3198,25 $\mu$ éq/l en saison sèche et $2168,98 \mu$ éq/l en saison pluvieuse. La présence marquée des nitrates dans les eaux de source du bassin versant du Nkié résulte des activités 
anthropiques, des activités agricoles et des effluents des fosses septiques (Barakatet al., 2018 ; Ahmed et al., 2019). Ils peuvent aussi provenir de la dégradation microbienne (minéralisation puis nitrification) de la matière organique et de l'oxydation de l'azote atmosphérique. En l'absence des roches évaporitiques dans le bassin, les ions chlorures pourraient avoir plusieurs origines : domestique (utilisation de l'hypochlorite de sodium), atmosphérique liée aux précipitations et par la présence du recouvrement argileux (Ahmed et al., 2019). Les sulfates quant à eux proviendraient de l'oxydation de la pyrite (Zianiet al., 2017) avec mise en solution du fer dans les formations gneissiques (Garrels, 1976) suivant l'équation :

$2 \mathrm{FeS}_{2}+15 / 2 \mathrm{O}_{2}+4 \mathrm{H}_{2} \mathrm{O} \longrightarrow \mathrm{Fe}_{2} \mathrm{O}_{3}+4 \mathrm{SO}_{4}^{2^{-}}+8 \mathrm{H}^{+}$(II). (Garrels, 1976).

Tableau 6. Paramètres physicochimiques et bactériologie des eaux de source du bassin versant du Nkié et Normes de l'OMS (2011).

\begin{tabular}{|c|c|c|c|}
\hline Paramètres & Saison sèche & Saison pluvieuse & Normes OMS \\
\hline Température $\left({ }^{\circ} \mathrm{C}\right)$ & 22 & 23,3 & - \\
\hline $\mathrm{pH}$ & 4,5 & 4,5 & 6,5 à 8,5 \\
\hline C.E $(\mu \mathrm{S} / \mathrm{cm})$ & 243 & 251 & - \\
\hline Chlorures $\mathrm{Cl}^{-}(\mathrm{mg} / \mathrm{l})$ & 59,27 & 22,25 & 35 \\
\hline Silice $\mathrm{SiO}_{2}(\mathrm{mg} / \mathrm{l})$ & 9,67 & 4,12 & - \\
\hline Sulfates $\left(\mathrm{SO}_{4}\right)^{2-}(\mathrm{mg} / \mathrm{l})$ & 1,74 & 0,3 & 400 \\
\hline Nitrates $\mathrm{NO}_{3}^{-}(\mathrm{mg} / \mathrm{l})$ & 92,53 & 93,37 & 40 \\
\hline Calcium $\mathrm{Ca}^{2+}(\mathrm{mg} / \mathrm{l})$ & 1,75 & 1,94 & 500 \\
\hline Magnésium $\mathrm{Mg}^{2+}(\mathrm{mg} / \mathrm{l})$ & 1,53 & 1,58 & 500 \\
\hline Fer $(\mathrm{mg} / \mathrm{l})$ & 0,12 & 0,26 & 0.3 \\
\hline Sodium $\mathrm{Na}^{+}(\mathrm{mg} / \mathrm{l})$ & 38,04 & 8,15 & 200 \\
\hline Potassium $\mathrm{K}^{+}(\mathrm{mg} / \mathrm{l})$ & 2,4 & 2,31 & - \\
\hline Bicarbonates $\mathrm{HCO}_{3}^{-}(\mathrm{mg} / \mathrm{l})$ & 0 & 1,83 & - \\
\hline Fluor (mg/l) & 1,59 & 0,06 & 1,5 \\
\hline $\mathrm{TZ}^{+}(\mu \mathrm{e} q / \mathrm{l})$ & 1928,72 & 640,47 & - \\
\hline TZ ( $\mu$ éq/l) & 3198,25 & 2168,98 & - \\
\hline Balance ionique (en \%) & $-24,76$ & $-54,41$ & - \\
\hline $\mathrm{SF}(\mathrm{UFC} / 100 \mathrm{ml})$ & 0 & 10 & absence \\
\hline CF (UFC/100ml) & 0 & 280 & absence \\
\hline
\end{tabular}

De la décomposition ou de la dégradation des êtres vivants (végétaux, animaux) et des apports atmosphériques.

Les concentrations en silice sont de $1,47 \mathrm{mg} / \mathrm{l}$ en saison pluvieuse et $3,453 \mathrm{mg} / 1$ en saison sèche. L'équation (I) montre que la production de la silice est liée à celle des cations et des bicarbonates. Les concentrations de silice obtenues sont liées à la nature lithologique silicatée du substratum rocheux.

La teneur en fer varie dans les eaux de source soit $0,26 \mathrm{mg} / \mathrm{l}$ en saison pluvieuseet $0,123 \mathrm{mg} / \mathrm{l}$ en saison sèche. Les valeurs obtenues sont inférieures à la valeur seuil $(0,3 \mathrm{mg} / \mathrm{l})$ recommandée par l'OMS (2011) pour la consommation humaine. Sa présence dans les eaux souterraines du bassin 
versant du Nkié serait liée à la dissolution de l'encaissant qui est une roche mère constituée de minéraux ferromagnésiens.

La représentation graphique de Piper montre que les eaux de source du bassin versant du Nkié appartiennent au faciès chloruré sodique et potassique ou sulfatées sodique (Figure 10). Cette typologie ( $\mathrm{NaK}-\mathrm{Cl}$ ou $\left.\mathrm{NaK}-\mathrm{SO}_{4}\right)$ se rapproche de celle trouvée par Nteuga (2012) dans le même bassin.

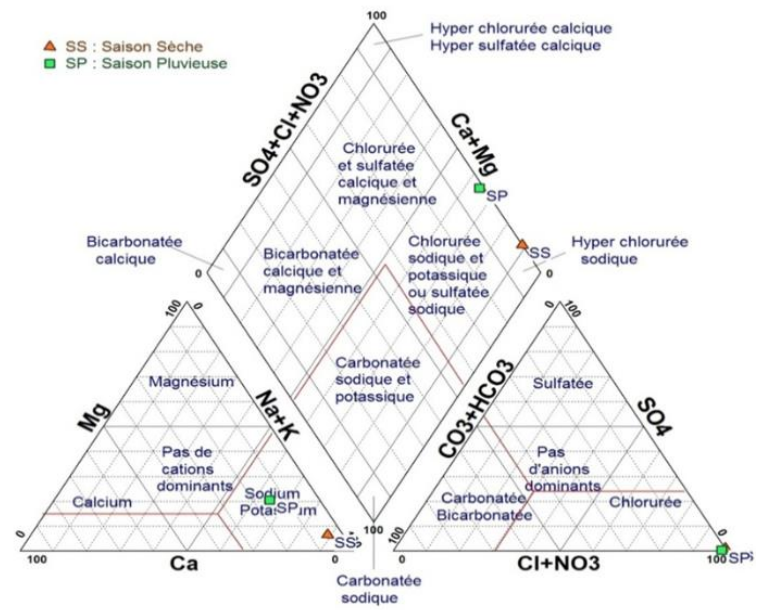

Figure 10. Diagramme de Piper (1944) des eaux de source du bassin versant du Nkié

Les coliformes fécaux (CF) et les streptocoques fécaux (SF) présentent des concentrations de $280 \mathrm{UFC} / 100 \mathrm{ml}$ et $10 \mathrm{UFC} / 100 \mathrm{ml}$ dans les eaux de source prélevées pendant la saison pluvieuse. Par contre, ces concentrations sont conformes en saison sèche à la norme OMS (2011) soit 0 UFC/100 ml. Elles traduisent ainsi le caractère pollué des eaux avec pour principales sources de contamination le rejet d'eaux usées domestiques, les débordements des réseaux d'égouts et l'épandage de fumier. Ces paramètres bactériologiques et même physicochimiques pris en compte dans le tableau 6 montrent que les eaux souterraines du bassin versant du Nkié ne sont pas de bonne qualité.La présence remarquée des nitrates $\left(\mathrm{NO}_{3}{ }^{-}<93,37 \mathrm{mg} / \mathrm{l}\right)$, des coliformes fécaux $(\mathrm{CF}<280 \mathrm{UFC} / 100 \mathrm{ml})$ et des streptocoques fécaux $(\mathrm{SF}<10 \mathrm{UFC} / 100 \mathrm{ml})$ dont les concentrations sont supérieures aux limites de toxicité définies par l'OMS (2011) induisent une pollution bactériologique et chimique de ces eaux. Ceci est dû à la mauvaise évacuation des eaux usées et des excréta. Ces eaux nécessitent donc à cet effet un traitement préalable avant toute consommation. Les coliformes et streptocoques fécaux observés en saison des pluies proviennent exclusivement des matières fécales des animaux à sang chaud (Rodier, 2009). Les coliformes et streptocoques fécaux sont de bons indicateurs de la présence potentielle d'organismes pathogènes tels que salmonella, shigella et vibrio cholerae (Tita, 2008). Ils sont à l'origine de plusieurs problèmes de santé à l'instar des gastro- 
entérites, dysenteries, choléra et dermatites. Les eaux de source du bassin du Nkié sont qualifiées de « moyenne » en saison pluvieuse et «très bonne » en saison sèche d'après Djeudaet al.,(1999), en référence à leur caractéristique bactériologique (Tableau 6). La présence des germes bactériologiques dans ces eaux indique qu'elles ne sont pas protégées de la prolifération d'une flore étrange (Nolaet al., 2001, 2002 ; Rodier, 2009). Ces eaux nécessitent pour ce fait un traitement obligatoire préalable avant leur consommation.

\section{Conclusion}

Le Bassin Versant (BV) du Nkié qui fait l'objet de la présente étude est situé au sud-est de la ville de Yaoundé. Il est l'un des 25 bassins que compte la ville de Yaoundé au Cameroun et est drainé par le cours d'eau principal Nkié. Le présent travail avait pour objectifs d'effectuer entre autres, une caractérisation physique des sols, hydrométrique des sources et hydrodynamique de l'aquifère à nappe libre, mais aussi une analyse des paramètres physico-chimiques et bactériologiques dans les eaux du BV du Nkié. Les principaux résultats sont les suivants :

1) la distribution granulométrique des produits d'altération révèle que chaque horizon est formé des grains de diamètres variés. Le coefficient d'uniformité (CU), est supérieur à 2,5 et les fractions fine et grenue des différents horizons sont respectivement comprises entre $24,69 \%$ et $95,31 \%$; et entre $4,69 \%$ et $75,31 \%$ illustrant le caractère varié de la granulométrie. Les teneurs en particules fines et grenues sont respectivement plus importantes dans l'horizon superficiel et dans le nodulaire.

2) On note cependant que les variations de ces débits suivent celles des précipitations. Il existe cependant un décalage entre les mises en charge de la nappe par rapport aux précipitations. Ce décalage témoigne d'une faible vitesse d'infiltration qui serait liée aux épaisses couches de sol, à l'état fissural et aux axes de drainage à direction variée. Une corrélation nonétablie entre les débits moyens annuels des sources et les altitudes révèle que les débits des sources ne sont pas liés à l'altitude du point de résurgence.

3) Les niveaux piézométriques varient entre 733,92 et $682,68 \mathrm{~m}$ pendant la période maximale, et entre 732,29 et 682,57 en période minimale. Les fluctuations piézométriques varient ainsi entre $0,1 \mathrm{~m}$ (zone de bas-fond) et 7,48 m (pour P3 situé au sommet des versants), soit une fluctuation moyenne annuelle de $1,36 \mathrm{~m}$. Les cartes piézométriques de l'aquifère à nappe libre montre un écoulement non uniforme des eaux souterraines. Cela est caractérisé sur les cartes par une direction variée des lignes de courant qui sont soit divergentes soit convergentes. Ces cartes révèlent ainsi des zones à arc de cercle concentrique portant des lignes de courant convergentes et celles à arc de cercle concentrique et portant des lignes de courant 
divergentes. Ces zones constituent respectivement des zones de stockage et d'alimentation de l'aquifère à nappe libre.

4) Le bilan hydrique révèle que l'aquifère à nappe libre se recharge d'avril à novembre (saison de pluies) et se vide progressivement de décembre à mars (saison sèche). Malgré la bonne pluviométrie de la ville de Yaoundé et de ses environs, la productivité des puits et sources restent modestes et baissent considérablement au fur et à mesure que l'on s'éloigne des axes des cours d'eau et/ou en sommet de versants.

5) Sur le plan de la potabilité, la présence des nitrates $\left(\mathrm{NO}_{3}{ }^{-}<93,37\right.$ $\mathrm{mg} / \mathrm{l})$, des coliformes fécaux $(\mathrm{CF}<280 \mathrm{UFC} / 100 \mathrm{ml})$ et des streptocoques fécaux $(\mathrm{SF}<10 \mathrm{UFC} / 100 \mathrm{ml})$ dont les concentrations sont supérieures aux limites de toxicité définies par l'OMS (2011) induisent une pollution bactériologique et chimique de ces eaux. L'origine de ces germes serait liée au rejet d'eaux usées domestiques non traitées, aux débordements des réseaux d'égouts et l'épandage de fumier. Ces eaux nécessitent donc à cet effet un traitement préalable avant toute consommation.

\section{References :}

1. Ahmed, I., Tariq, N., Al Muhery, A. (2019). Hydrochemical characterization of groundwater to align with sustainable development goals in the Emirate of Dubai, UAE. Environmental Earth Sciences 78:44. https://doi.org/10.1007/s12665-018-8030-1

2. Akrong, M, Amu-Mensah, F. K., Amu-Mensah M., Darko, H., Addico, G., Ampofo, J. (2019). Seasonal analysis of bacteriological quality of drinking water sources in communities surrounding Lake Bosomtwe in the Ashanti Region of Ghana. Applied Water Science 9:82. https://doi.org/10.1007/s13201-019-0959-z

3. Ameen-Ameen, H. (2019). Spring water quality assessment using water quality index in villages of Barwari Bala, Duhok, Kurdistan Region, Iraq. Applied Water Science 9:176. https://doi.org/10.1007/s13201-019-1080-z

4. An, T.D., Tsujimura, M., Le-Phu, V., Kawachi, A., Ha, D.T. (2014). Chemical characteristics of surface water and groundwater in coastal watershed, Mekong Delta, Vietnam. Procedia Environ Sci 20:712721. https://doi.org/10.1016/j.proenv.2014.03.085

5. Apha, (2012). Standard methods for examination of water and wastewater, 22nd edn. American Public Health Association, Washington. ISBN: 978-087553-013-0

6. Barakat, A., Meddah, R., Afdali, M., Touhami, F. (2018). Physicochemical and microbial assessment of spring water quality for drinking supply in Piedmont of Béni-Mellal Atlas (Morocco). Phys Chem Earth Parts A/B/C 104:39-46 
7. Bon, A.F. (2008). Hydrodynamique d'un bassin versant en zone de socle cristallin fracturé et alteré : cas du bassin versant de l'Olezoa au Sud - Ouest de la ville de Yaoundé - Cameroun. Mém. DEA. Univ Yaoundé I, Fac Sci. Dpt Sciences de la Terre. 78 p.

8. Bosso-Bosso, S. (2000). Étude hydrogéologique d'une zone urbaine de socle : cas de la ville de Yaoundé - Cameroun. Mém. DEA. Fac. Sci. Univ. Yaoundé I, 41p.

9. BUCREP, (2010). Troisième recensement général de la population et de l'habitat, $67 \mathrm{p}$.

10. Castany, G. (1998). Principes et méthodes de l'hydrogéologie. Éd. Dunod, Paris V, 236 p.

11. Collin, J.J. (2004). Les eaux souterraines. Connaissance et gestion. Éd. BRGM, 174p. Communauté Urbaine de Yaoundé, 2007. Planification urbaine au Cameroun : cas de la ville de Yaoundé $31 \mathrm{P}$.

12. Djeuda-Tchapnga, H.B., Tanawa, E., Ngnikam, E. (1998). Contraintes sociales liées à la mise en place des périmètres de protection des ressources en eau dans les zones périurbaines et les petits centres des pays en développement. Communication présentée au IIème symposium international en gestion et technologies appropriées pour l'eau aux petits ensembles habités. Barcelone (Espagne). Du 13 au 15octobre 1998, 11 p.

13. Djeuda-Tchapnga, H.B., Tanawa, E., Ngnikam, E. (2001). L'eau au Cameroun : Tome 1 : Approvisionnement en eau. Éd. Press. Univ.de Yaoundé, $356 \mathrm{p}$.

14. Djeuda-Tchapnga, H.B., Tanawa, E., Temgoua, E., Siakeu, J., NgoMassana, B. (1999). Modèles de circulation, mécanismes de recharge et temps de séjour des eaux souterraines des altérites en milieu cristallin : cas du bassin versant de l'Anga'a, Yaoundé - Cameroun. Collect. GÉOCAM, 2/1999, Éd. Sci. : VICAT J.P., BILONG P., Éd. Presses. Univ. Yaoundé I, pp 117 - 126.

15. Ekodeck, G.E. (1984).Altération des roches métamorphiques du SudCameroun et aspects géotechniques. Th. Doc. d'Etat. Univ. Grenoble, $368 \mathrm{p}$.

16. Ekodeck, G.E., Kamgang-Kabeyene, B.V. (2002). L'Altérologie normative et ses applications. Une expression particulière de la pétrologie des roches aluminosilicatées du point de vue de leur évolution supergène. Press. Univ. Yaoundé, $231 \mathrm{p}$.

17. Eshtawi, T., Evers, M., Tischbein, B. (2016). Quantifying the impact of urban area expansion on groundwater recharge and surface runoff. Int Assoc Sci Hydrol Bull 61(5):826843.https://doi.org/10.1080/02626667.2014.1000916 
18. Feumba, R. (2005). Hydrogéologie en zone de socle cristallin : Cas du bassin versant de l'Ekozoa, secteur nord de la ville de Yaoundé Cameroun. Mém. DEA. Fac. Sci. Univ. Yaoundé I, 94 p.

19. Fouepe-Takounjou, A., Ndam-Ngoupayou, J.R., Marechal, J., Riotte, J., Sigha-Nkamdjou L., Mafany, Ekodeck, G.E. (2011). Estimation of groundwater recharge of shallow aquifer on humid environment in Yaoundé, Cameroon using hybrid-water fluctuation and hydrochemistry methods. Environmental Earth Sciences. 12p.

20. Garrels, R.M. (1976). A Survey of Low Temperature Water Mineral Relations. In: Interpretation of Environmental Isotope and Hydrogeochemical Data in Groundwater Hydrology, International Atomic Energy Agency, Vienna, 65-84.

21. Hem, J.D. (1985). Study and interpretation of the chemical characteristics of natural water, vol 2254. Department of the Interior, US Geological Survey, Reston

22. Kalla-Mpako, F. (2007).Caractérisation physique et hydrodynamique de l'aquifère à nappe libre du bassin versant de Ntem à YaoundéCameroun. Mém. DEA. Fac. Sci. Univ. Yaoundé I, 99 p.

23. Kendzo-Nguefack, J. F. (2008). Etude piézométrique, Hydrochimique et bactériologique des nappes en zone de socle cristallin : cas du bassin versant de Ntsomo (Yaoundé - Cameroun). Mém. DEA. Univ Yaoundé I, Fac Sci. Dpt Sciences de la Terre. 72p.

24. Kouam, K.G.R., Mpakam, H.G., Ewodo, M.G., Kwenkeu, Tchabo, M., Bayiga, E., Ngoutié, M., Mabou, K.M. (2006). Rapport d'activité de la 13e semaine nationale de l'eau (15 au 22 mars 2006) et de la $14 \mathrm{e}$ journée mondiale de l'eau (22 mars 2006) sous le thème « eau et culture » p.73.

25. Kuate-Defo, T. H. (2008). Hydrodynamique des nappes en zone de socle cristallin du bassin versant de la Biyemé à Yaoundé Cameroun. Mém. DEA. Univ Yaoundé I, Fac Sci. Dpt Sciences de la Terre. 69p.

26. LESEAU, (2004). Maîtrise de l'assainissement dans un écosystème urbain à Yaoundé au Cameroun et impact sur la santé des enfants âgés de moins de cinq (5) ans. Rapport de recherche de l'année 2003/2004, ENSP de Yaoundé.

27. Ma, F.S., Wei, A.H., Deng, Q.H., Zhao, H.J. (2014). Hydrochemical characteristics and the suitability of groundwater in the coastal region of Tangshan, China. J Earth Sci 25(06):10671075.https://doi.org/10.1007/s12583-014-0492-9

28. Melo, A., Pinto, E., Aguiar, A., Mansilha, C., Pinho, O., Ferreira, I. (2012). Impact of intensive horticulture practices on groundwater content of nitrates, sodium, potassium, and pesticides. Environ Monit Assess 184 :4539-4551. https://doi.org/10.1007/s10661-011-2283-4 
29. MINEE-GWP, (2009). Plan d'Action National de Gestion Intégrée des Ressources en Eau: Etat des lieux du secteur de l'eau au Cameroun, p 213.

30. Mpesse, J.E. (1999). Contribution à l'étude pétrostructurale des formations métamorphiques de la région de Yaoundé et de la formation de la géométrie de sa tectonique tangentielle. Th. Doc. 3e cycle, Univ. Yaoundé I, 148 p. Mer. 9 Edition, Dunod, Paris, 1382 pp.

31. Nola, M., Njiné, T., Djuikom, E., Sikati F.V. (2002). Faecal coliforms and faecal streptococci community in the underground water in an equatorial area in Cameroon (Central Africa): the importance of some environmental chemical factors. Water Res. 36: 3289-3297.

32. Nola, M., Njiné, T., Sikati, F.V., Djuikom, E. (2001). Distribution de Pseudomonas aeruginosa et Aeromonas hydrophila dans les eaux de la nappe phréatique superficielle en zone équatoriale au Cameroun et relations avec quelques paramètres chimiques du milieu. Rev. Sci. Eau 14: 35-53.

33. Ntep, F.(2005). Hydrodynamique et qualité des eaux des nappes en zone de socle cristallin fissuré et altéré : Cas du bassin versant de la Mingoa (Yaoundé - Cameroun). Mém. DEA. Fac. Sci. Univ. Yaoundé I, $84 \mathrm{p}$.

34. Nteuga-Njike S. (2012). Fonctionnement Hydrologique et Qualité des eaux du bassin versant du Nkié à Yaoundé (Sud-Cameroun). Mém. Master, Fac. Sci. DST. Univ. Yaoundé I, 57p.

35. Olivry, J.C. (1986). Fleuves et rivières du Cameroun. Monographies hydrologiques, MESRES/ORSTOM, nº 9, 733 p.

36. OMS (2011). Guidelines for drinking-water quality recommendations. Fourth edition. WHO, Geneva,p 518

37. Onana, V.L. (2001). Caractérisation et intérêts géotechniques des produits d'altération des roches métamorphiques du secteur Mengang-Mfoumasi (région de Yaoundé-Cameroun). Mém. Maît. Fac.Sci. Uni. Yaoundé I. 81p.

38. Parriaux, A., Bussard, T. (2002). Dimensionnement des aires d'alimentation Zu. OFEFP, Lausanne, 143 p.

39. Piper, A.M. (1944). A graphic procedure in the geochimical interpretation of water analyses. Americain Geophysical Union, Transcript 25, 914-923.

40. Porchet, M. (1931). Hydrodynamique des puits. Ann. du Génie Rural, fasc. 60.

41. Priso-Bile F. (2007). Etude des caractéristiques physiques, hydrodynmiques et de la vulnérabilité de l'aquifère à nappe libre du 
bassin versant de l'Ebogo - Ewe à Yaoundé. Mém. DEA, Fac. Sci. DST. Univ. Yaoundé I. 67p.

42. Remenieras, G. (1972). Hydrologie de l'ingénieur. 3e édition. Eyrolles (collection du centre de recherches et d'essais de Chatou), 454 p.

43. Rodier, J. (2009). L'analyse de 1'Eau ; Eau Naturelle, Eau Résiduaire, Eau de Mer. Doc., tech. 9e édn. Dunod (Ed) :Paris.

44. Selvam, S., IruthayaJeba Dhana Mala R., Muthukakshmi V. (2013). A hydrochemical analysis and evaluation of groundwater quality index in Thoothukudi district, Tamilnadu, South India. Int J Adv Eng Appl 2(3):25-37

45. Sighomnou, D. (2004). Analyse et redéfinition des régimes climatiques et hydrologique. Perspectives d'évolution des ressources en eau. Th. Doc d'Etat. Univ. Yaoundé I. 289p.

46. Suchel, J. B. (1987). Les climats du Cameroun. Thèse Doctorat 3e cycle Univ. Bordeaux III 186 p.

47. Tanawa, E., Djeuda-Tchapnga, H. B., Ngnikam, E., Temgoua, E., Siakeu, J. (2000). Habitat and protection of water resources in suburban areas in Africa cities. Building and Environment. 37, Pergamon, Elsevier. 269-275.

48. Tanawa, E., Djeuda-Tchapnga, H.B., Ngnikam, E., Temgoua, E., Siakeu, J. (2001). Habit and protection of water resources in suburban areas in African cities. BAE 545; pp $1-7$.

49. Tanawa, E., Djeuda-Tchapnga, H.B., Ngnikam, E., Temgoua, E., Siakeu, J. (2003). Habitat and protection of water resources in suburban areas in Africa cities. Build in gand Environment 37. pp. 269 - 275. Elsevier (Ed). Pergamon.

50. Thornwaite, C.W. (1954). The measurement of potential evapotranspiration, John p. Mather Seebrook, New Jersey, 222 p.

51. Tita, M.A. (2008). Water pollution of the Nkoup river system and its environmental impact on Foumbot town (Western Cameroon). Thèse de Doctorat/phD, Univ. Ydé I 62p.

52. Turgeon, P., Michel, P., Levallois, P., Archambault, M., Ravel, A. (2011). Fecal Contamination of Recreational Freshwaters: the Effect of Time-Independent Agroenvironmental Factors. Water Qual Expo Health 3, 109. https://doi.org/10.1007/s12403-011-0048-5

53. UNESCO, (2003). «Faits et Chiffres : Approvisionnement en eau et assainissement », « http:/:www.wateryear2003.org ».

54. Ziani, D., Abderrahmane, B., Boumazbeur, A., Benaabidate, L. (2017). Water quality assessment for drinking and irrigation using major ions chemistry in the Semiarid Region: case of Djacer Spring, Algeria. Asian J Earth Sci 10:9-21 